\title{
Topography Measurement for Monitoring Manufacturing Processes in Harsh Conditions
}

\author{
Thomas Mueller, Andreas Poesch, Eduard Reithmeier \\ Institute of Measurement and Automatic Control, Leibniz University Hannover, Hannover, Germany \\ Email: thomas.mueller@imr.uni-hannover.de
}

Received 6 April 2016; accepted 20 May 2016; published 23 May 2016

Copyright (C) 2016 by authors and Scientific Research Publishing Inc.

This work is licensed under the Creative Commons Attribution-NonCommercial International License (CC BY-NC).

http://creativecommons.org/licenses/by-nc/4.0/

(c) (1) (8) Open Access

\begin{abstract}
High precision manufacturing, e.g. milling and grinding, which have manufacturing tolerances in the range of $<10 \mu \mathrm{m}$ require microscopic measurement techniques for the inspection of the manufactured components. These measurement techniques are very sensitive to cooling liquids and lubricants which are essential for many manufacturing processes. Therefore, the measurement of the components is usually conducted in separate and clean laboratories and not directly in the manufacturing machine. This approach has some major drawbacks, e.g. high time consumption and no possibility for online process monitoring. In this article, a novel concept for the integration of high precision optical topography measurement systems into the manufacturing machine is introduced and compared to other concepts. The introduced concept uses a reservoir with cooling liquid in which the measurement object is immersed during the measurement. Thereby, measurement disturbance by splashing cooling liquids and lubricants can effectively be avoided.
\end{abstract}

\section{Keywords}

Optical Inspection, Topography Measurement, Laser Triangulation, Precision Manufacturing

\section{Introduction}

In high precision manufacturing processes, e.g. milling, turning, grinding, it is often necessary to measure the topography of the components in order to monitor the accuracy of the manufacturing process. Confocal microscopy, white light microscopy and laser triangulation are examples for suitable measurement techniques for monitoring manufacturing process accuracies in the range of $<10 \mu \mathrm{m}$. These commercially available measure- 
ment systems can determine the surface topography of components with a measurement uncertainty of $<1 \mu \mathrm{m}$ and, therefore, are able to identify components which do not comply with the manufacturing tolerances. However, most of the commercially available measurement systems for microscopic topography measurements are very sensitive to any disturbance during the measurement, for example vibrations and splashing cooling liquids. Since a large number of manufacturing processes, e.g. milling and grinding, require the use of cooling liquids and lubricants, microscopic topography measurement can usually not be performed inside the manufacturing machine. Splashing liquids and lubricants can damage or destroy high precision microscopes and even when the microscope is installed in a protective housing, the measurement process is disturbed by cooling liquids and lubricants. Figure 1 shows the comparison of an undisturbed microscopic measurement and a measurement when there is a thin oil film between the microscope and the measurement object. The thickness of the oil film is $<100$ $\mu \mathrm{m}$, however, it prevents the microscope from obtaining sharp images of the measurement object. This means that even very thin depositions of cooling liquids and lubricants on the viewing windows of the microscope's protective housing prevent the microscope from measuring the surface topography with accuracy in the micrometer range. Therefore, microscopic inspection is usually performed off-line in a separate and clean laboratory and not inside the manufacturing machine. The disadvantage of this approach is that not all manufactured components can be measured, because the transport of the component to the laboratory and the cleaning and preparation of the component before the measurement take a lot of time. Another important disadvantage is that this approach only allows assessing the manufacturing process at random. When the measurement shows that the component does not comply with the acceptable manufacturing tolerances, one or more components usually have to be disposed of, because subsequent machining is not possible. The reason for this is that after removing the component from the manufacturing machine, it is usually not possible to mount the component again in the exact same position as it was before which is required for a component processing accuracy in the range of $<10$ $\mu \mathrm{m}$. In order to avoid the disadvantages of separate laboratory measurements, a measurement inside the manufacturing machine is desired. In this case, the component can remain in the mounting of the manufacturing machine which shortens the duration of the measurement and allows for a subsequent processing of the component until it complies with the manufacturing tolerances. Precision component topography measurements have already been shown for manufacturing processes which do not cause measurement disturbance such as splashing cooling liquids [1]-[3]. In this paper, a novel approach for topography measurements in manufacturing machines with a high degree of disturbances due to liquids and material deposition is presented. For the topography measurement of the component, laser triangulation is used but the presented approach can be used for any optical topography measurement technique.

\section{Basic Setup of the Measurement System}

The basic setup of the developed measurement system for the topography measurement in the manufacturing machine is shown in Figure 2. In the presented application, the measurement system monitors the dressing of microstructures on a grinding wheel. Laser triangulation was chosen for the measurement of the topography. The measurement system includes a laser whose output light is focused on the surface of the grinding wheel by beam shaping optics. By the use of a cylindrical lens, the measurement object is illuminated with a laser line and

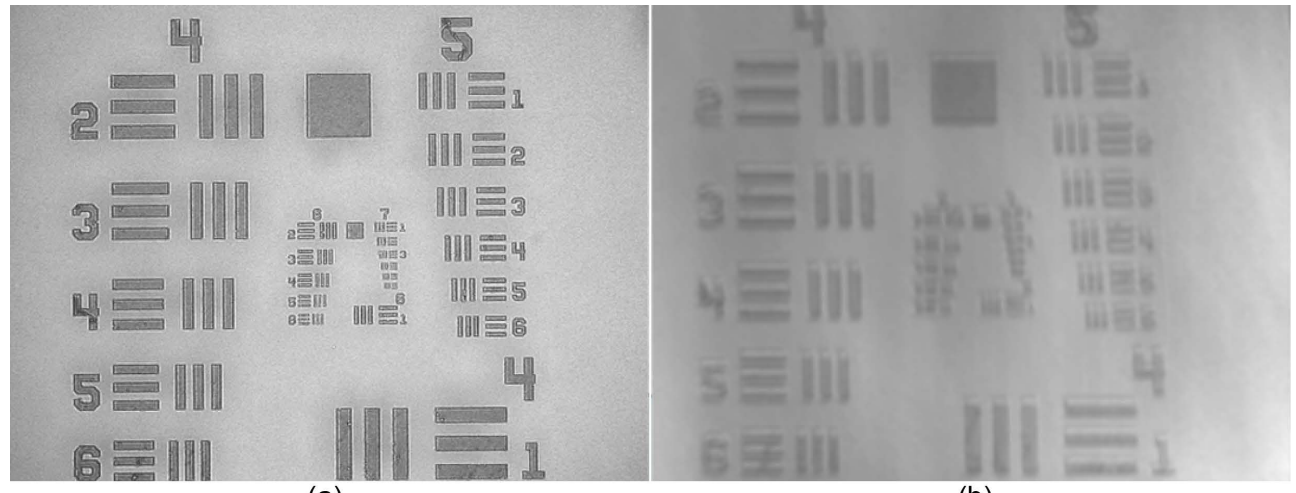

(a)

(b)

Figure 1. Measurement of a USAF1951 test target with a confocal microscope $(10 \times$ objective, NA $=0.3)$. (a) Measurement in standard operating conditions. (b) Measurement with a thin oil film between the microscope and the test target. 


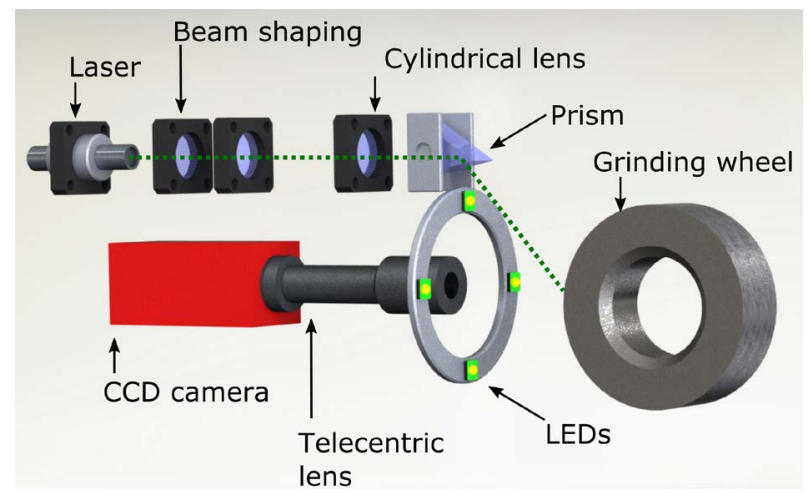

Figure 2. Basic setup of the measurement system.

not only with a laser spot. Therefore, the surface topography $z$ can be obtained in the form $z(x)$. The laser line on the component's surface is imaged by a telecentric lens with an adaptable magnification in the range of $2<\beta^{\prime}<10$. Details on laser triangulation can be found, for example, in [4]-[6]. The working principle of laser triangulation depends on the determination of the laser line shift $\Delta y$ in the camera image which is, then, used to calculate the surface topography. The calculation of the topography $z$ from the line position deviation $\Delta y$ in the camera image depends on the particular setup of the triangulation system. In [7], the topography is calculated by

$$
z=\frac{\Delta y \cdot f}{\left(x-f \sqrt{1+\left(\frac{l_{T}}{f}-\frac{1}{\tan (\alpha)}\right)^{2}}\right) \sin (\alpha)} .
$$

$f$ is the focal lens of the objective, $\alpha$ is the triangulation angle and $l_{T}$ is the triangulation base length. When a CCD matrix camera sensor is used, the laser line position $\Delta y$ needs to be calculated from the captured images. A good comparison of calculation algorithms can be found in [8]. A simple and effective algorithm is to calculate the image intensity center of gravity

$$
y=\frac{\sum_{x, y} y I(x, y)}{I(x, y)} .
$$

$I(x, y)$ is the intensity in the camera pixel $(x, y)$. Details on the measurement uncertainty of laser triangulation can be found in [6] and [9]. A fundamental limit of laser triangulation is caused by speckle which is a patterned redistribution of the laser light intensity on the measurement object's surface due to interference effects of the coherent laser light. In [6] the uncertainty $\delta y$ of the laser line position calculation by speckle is determined using

$$
\delta y=\frac{1}{2 \pi} \frac{1}{\sin (N A)} .
$$

$N A$ is the numerical aperture of the imaging lens.

An advantage of laser triangulation is that it is suitable for the measurement of fast rotating and moving components. Pulsed lasers with very high output light intensities and cameras with very high frame rates in the range of 10,000 - 100,000 images per second are commercially available. Another advantage of laser triangulation is that the measurement accuracy can also be adapted depending on the particular measurement task. This can be achieved, for example, by changing the camera lens to a different magnification or by adapting the triangulation angle $\alpha$. For the presented application in this paper, the CCD camera "AVT Pike 421B" and the telecentric lens "Sill Optics S5LPJ0455" is used. The camera has a resolution of $2048 \times 2048$ pixels and a pixel size of 7.4 $\mu \mathrm{m}$. The telecentric lens has an adaptable magnification $\left(2<\beta^{\prime}<10\right)$ and an adaptable aperture. It should be noted, that the presented concept for the integration of the measurement system into the manufacturing machine is not limited to these components and also not limited to laser triangulation. 


\section{Measurements in the Manufacturing Machine}

\subsection{Effects of Cooling Liquids and Lubricants}

The basic measurement system setup, which is shown in Figure 2, needs to be installed in a housing in order to protect the sensitive components from the splashing cooling liquids. A photo of the manufacturing machine of the presented application is given in Figure 3 and Figure 4 shows the machine during component processing. It can be seen that the cooling liquid is distributed in the entire processing chamber of the machine as a splashing liquid and as a liquid mist. Therefore, the viewing windows of the measurement system's housing are always covered by cooling liquid during component processing. The effects of cooling liquid on the viewing window for the camera and objective are demonstrated in Figure 1 and Figure 5 shows the effects on the laser line. When the viewing window is covered with the cooling liquid, no sharp laser line is obtained and therefore the topography cannot be measured with sufficient measurement accuracy. It is also important to consider the effects of cooling liquids on the measurement object. Figure 6 shows the laser line on the surface of a measurement object which topography shows a periodic sinus-shaped pattern. Here, no oil film is present on the surface for the (a) image. For the (b) and (c) image, a thin oil film was disposed on the measurement object.

The dashed lines in Figure 6 indicate the upper and lower limits of the laser line for the undisturbed measurement ((a) image). In the (b) and (c) measurement, the lower limit of the laser line does not coincide with the undisturbed measurement. The oil film on the measurement object's surfaces interferes with the propagation path of the laser light. Therefore, the topography of the measurement object, in particular the amplitude of the sinus-pattern, is not correctly measured. The influence of the oil film on the measurement object's surface is more critical than the influence of an oil film on the viewing window. The shape of the laser line with oil on

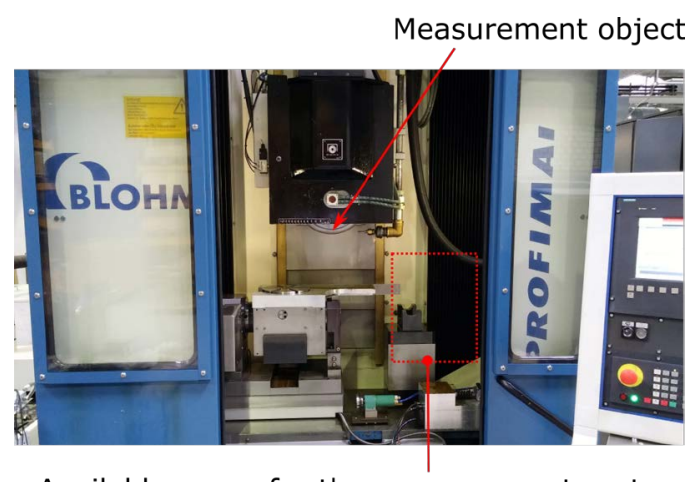

Available space for the measurement system

Figure 3. Manufacturing machine in which the measurement system is installed.

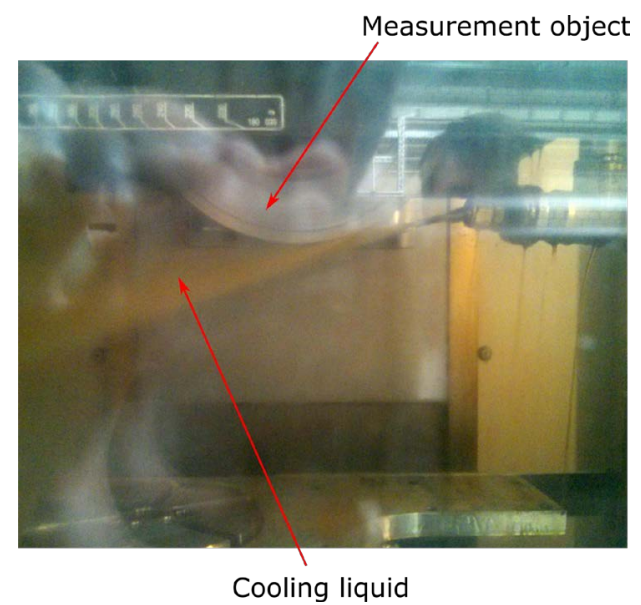

Figure 4. Active manufacturing machine during component processing (blurred due to cooling liquid). 


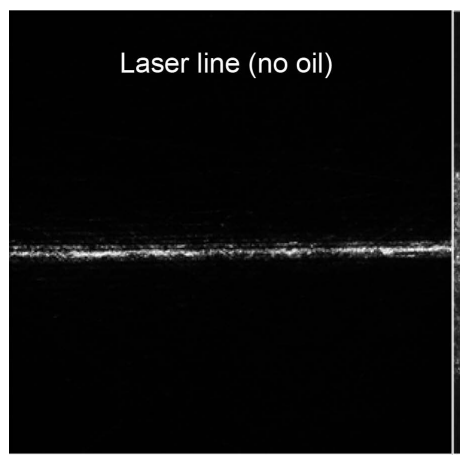

(a)

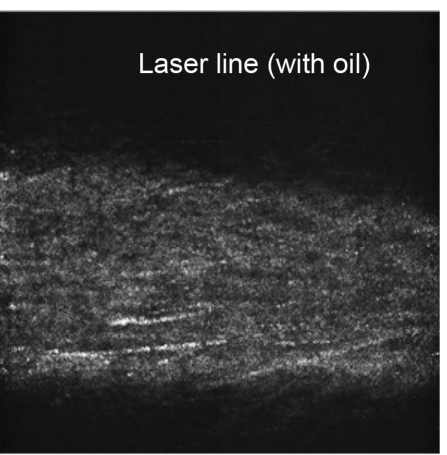

(b)

Figure 5. Laser line on the measurement object's surface without (a) and with (b) oil on the viewing window.

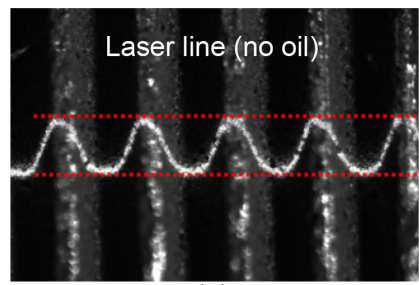

(a)

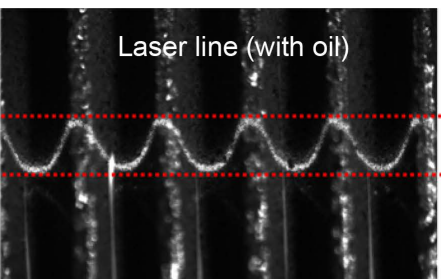

(b)

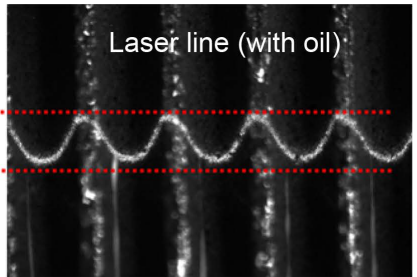

(c)

Figure 6. Laser line on the measurement object's surface. (a) No oil film on the measurement object. (b) and (c) Thin oil film on the measurement object's surface.

the viewing window clearly differs from the undisturbed case. Thus, influenced measurements can be identified by automated image processing and the measurement data can be rejected for topography calculation until the laser line is not disturbed. However, the influence of an oil film on the measurement object cannot be identified from the measurement data. In Figure 6, the appearance of the laser line does not seem to be significantly different for the disturbed and not disturbed cases. Therefore, disturbed measurements cannot be identified and wrong measurement data of the surface topography is obtained.

\subsection{Concepts for the Integration}

As described in the previous section, the effect of cooling liquids and lubricants must be avoided in order to obtain undisturbed topography measurements. Three concepts for the integration of the measurement system into the manufacturing machine were evaluated:

- Concept A: Permanent removal of the cooling liquids, lubricants or other pollutants from the viewing window and from the measurement object using high pressured air.

- Concept B: Similar to concept A, but no removal of liquids from the viewing window of the camera. Therefore, the setup is compacter and uses less compressed air. The effects of the liquids on the camera images are reduced using blind deconvolution image processing algorithms.

- Concept C: No removal of the cooling liquid. For the execution of a measurement, the measurement object is immersed in a liquid reservoir which is mounted on the viewing window of the camera and laser line projector.

Concept A is illustrated in Figure 7. Nozzles for compressed air are directed to the viewing windows of the camera and laser line generator and the measurement object. The camera with telecentric lens and the laser line generator are installed in separate housings which makes the adjustment of the optical components easier when the measurement system is installed in the manufacturing machine.

Concept B is a similar approach to concept A but without the compressed air nozzle for the viewing window of the camera. Thus, the cooling liquid and other pollutants are not removed from this viewing window. However, it can be attempted to avoid the disturbances by blind deconvolution image processing algorithms. The disturbed image $I_{a b e r}^{\prime}(x, y)$ can be considered as a convolution of the undisturbed image and a local varying 


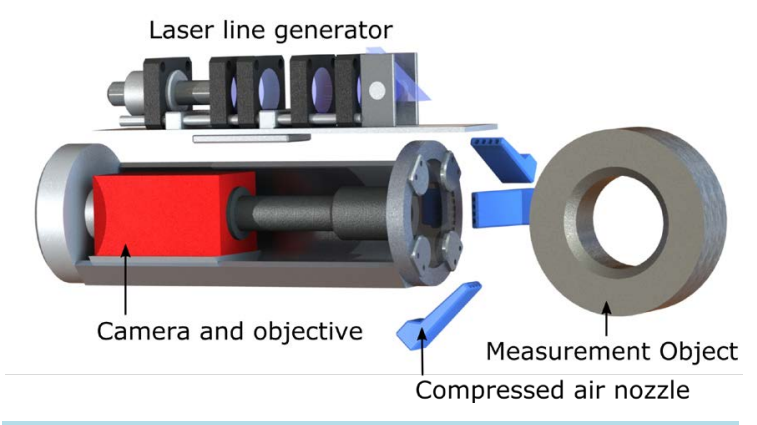

Figure 7. Integration using concept A.

point spread function

$$
I_{\text {aber }}^{\prime}(x, y)=P S F_{a b e r} * I^{\prime}(x, y)
$$

The point spread function $P S F_{a b e r}$ depends on the thickness and refractive index of the liquid film on the viewing window. Usually, this point spread function is not known, but blind deconvolution image processing algorithms can be used to estimate this function. Examples of suitable algorithms can be found in [10]-[12].

The compressed air nozzles for the viewing window of the laser projector and for the measurement object are still necessary because the influence of cooling liquids on these components cannot be reduced by image processing algorithms. During the evaluation of suitable algorithms [13] [14], the obtained reliability was not sufficient. The reason for this is, that the liquids on the viewing window can cause many different aberrations, e.g. image rotation, displacement, blurring and double images. Most of the blind deconvolution algorithms work well for some specific aberrations, which is in most cases images blurring. Therefore, the point spread function is correctly estimated only in a few cases, when mainly image blurring is present. In other cases, the estimation is not correct and the image is not correctly reconstructed. Therefore, concept B was not chosen for the integration of the measurement system in the manufacturing machine.

Concept $\mathrm{C}$ uses a completely different approach compared to concept A and B. It is not attempted to remove the cooling liquid from the viewing windows and the measurement object. Figure 8 shows the assembly of the concept. The camera with objective and the laser line generator are installed in a common protective housing and have a shared viewing window. A reservoir containing a liquid is installed on top of the viewing window. During a measurement, the measurement object is immersed in the reservoir. The surface of the measurement object is illuminated and imaged through a layer of the liquid in the reservoir. For highest robustness, the liquid in the reservoir should be the same as the cooling liquid, lubricant or any liquid which is mainly present in the manufacturing machine. Thereby, disturbance by mixing two liquids with different optical properties in the reservoir is avoided. Concept $\mathrm{C}$ is very robust towards the distribution of cooling liquids and lubricants in the manufacturing machine. Due to the immersion of the measurement object, the measurement conditions remain constant for each measurement and the influence of the splashing liquids and mist are avoided. However, concept $\mathrm{C}$ has the disadvantage that the optical triangulation for the surface topography measurement is no performed in air but in a liquid which has a refractive index $>1$. For example, the cooling liquid which was used in the experiments performed for this article, has a refractive index of $n=1.46$. The laser beam is refracted at the boundary layer of the liquid towards a smaller angle relating to the optical axis of the camera and objective. This means that the triangulation angle is reduced which generally means a reduced sensitivity $\mathrm{d} y / \mathrm{d} z$ of the laser triangulation. For example, a refractive index of $n=1.46$ reduces a triangulation angle from $\alpha_{\text {air }}=45^{\circ}$ to $\alpha_{n=1.46}=29^{\circ}$.

\subsection{Evaluation in the Manufacturing Machine}

Concept A and concept C are both suited for the measurement of the component's surface topography in the manufacturing machine. Therefore, both concepts were installed in the manufacturing machine which is shown in Figure 3. In Figure 9 the installation of concept $A$ and in Figure 10 the installation of concept $C$ is shown. It can be seen, that the setup of concept $C$ is compacter than concept $A$ because no external compressed air nozzles and pipes to the air compressor are required. Thereby, concept $\mathrm{C}$ is more versatile and can be mounted faster. The deciding factor is reliability which is much better for concept $\mathrm{C}$. When using concept $\mathrm{A}$, the pollutants are removed in the majority of the cases by the compressed air, however in some cases, a small film of the cooling 


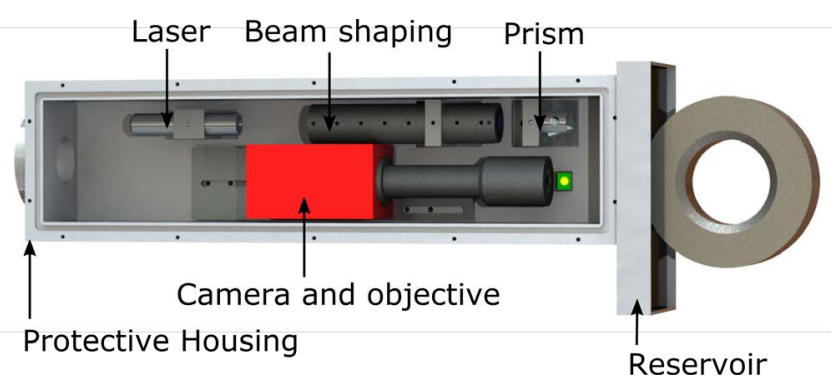

Figure 8. Assembly of concept C. For better visualization in the figure, the measurement system is rotated $90^{\circ}$ compared to the installation position in the manufacturing machine.
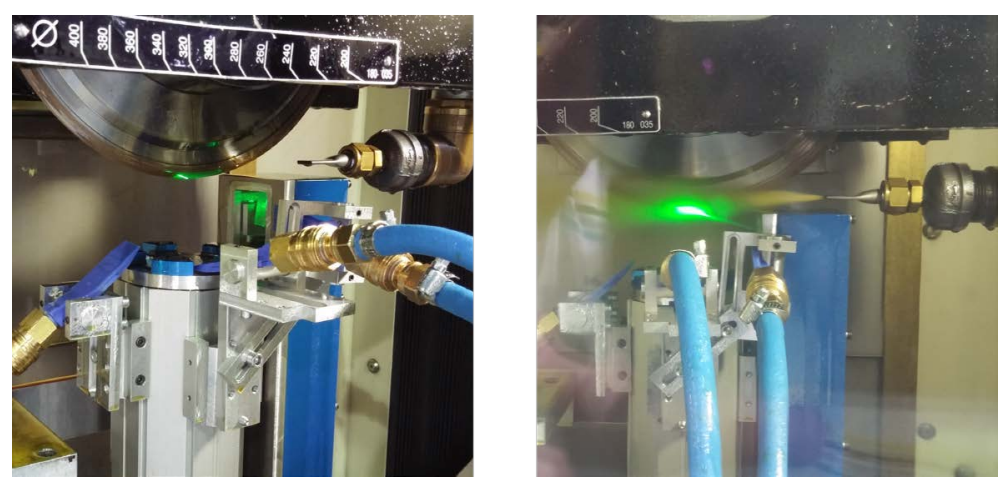

Figure 9. Installation of concept A in the manufacturing machine.

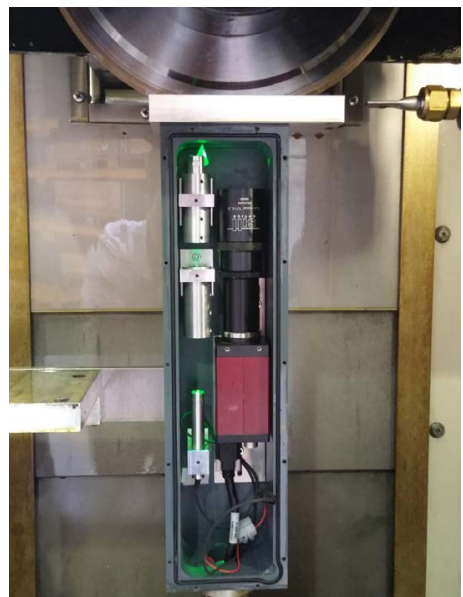

Figure 10. Installation of concept $\mathrm{C}$ in the manufacturing machine.

liquid remains on the viewing windows. In these cases, the manufacturing process needs to be interrupted for the cleaning of the viewing windows. When using concept $\mathrm{C}$, the measurement conditions remained constant in all measurements and they were not disturbed by splashing liquids or mist. Therefore, we advise to use concept $\mathrm{C}$ for the integration of high precision topography measurement systems in manufacturing machines.

\subsection{Measurement Results Using Concept C}

A measurement of a microstructured surface through the cooling liquid reservoir is shown in Figure 11. It can be seen that sharp and clear images can be obtained even when the measurement is performed through a layer of cooling liquid which has a dark brown color (see Figure 4). The thickness of the cooling liquid was approximately $3 \mathrm{~mm}$. 


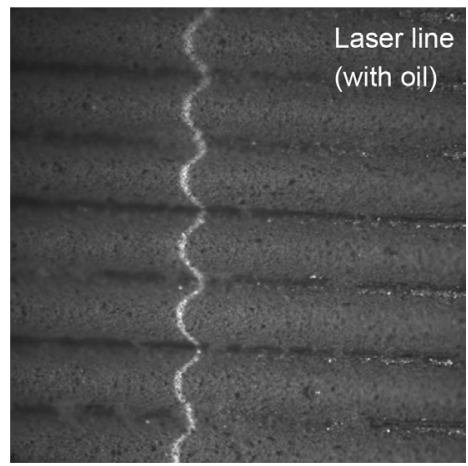

Figure 11. Laser triangulation measurement of a microstructured surface using concept $\mathrm{C}$ in the manufacturing machine.

In some manufacturing machines, not only splashing cooling liquids, lubricants and mist can occur, but also material which was ablated from the component due to the manufacturing process. This material can accumulate in the reservoir and can block the clear view of the laser line projector and camera to the measurement object. In these cases, the reservoir can be upgraded with cooling liquid pipes to allow the cooling liquid to run through the reservoir to a filter. Thereby, no material can accumulate in the reservoir and the views of the camera and laser line generator remain undisturbed.

\section{Conclusion}

In this article, a new concept is introduced for the topography measurement of components in manufacturing machines which have a high degree of splashing cooling liquids, lubricants and mist. Usually, high precision measurements are not possible in these operating conditions. In the introduced concept, the measurement object is immersed in a reservoir of cooling liquid and the measurement is conducted through the cooling liquid. Thereby, the measurement conditions remain constant at all times, and the topography can reliably be measured even in these harsh operating conditions.

\section{Acknowledgements}

This publication is based on a venture, referenced by project code 03V0473, which is sponsored by the Federal Ministry of Education and Research (BMBF). The authors are responsible for the content of this publication.

\section{References}

[1] van der Bijl, R.-J.M., Fähnle, O.W., van Brug, H. and Braat, J.J.M. (2000) Inprocess Monitoring of Grinding and Polishing of Optical Surfaces. Applied Optics, 39, 3300. http://dx.doi.org/10.1364/AO.39.003300

[2] Fan, K.-C., Lee, M.-Z. and Mou, J.-I. (2002) On-Line Non-Contact System for Grinding Wheel Wear Measurement. International Journal of Advanced Manufacturing Technology, 19, 14-22. http://dx.doi.org/10.1007/PL00003964

[3] Lim, H.S., Son, S.M., Wong, Y.S. and Rahman, M. (2007) Development and Evaluation of an On-Machine Optical Measurement Device. International Journal of Machine Tools and Manufacture, 47, 1556-1562. http://dx.doi.org/10.1016/j.ijmachtools.2006.11.007

[4] Ji, Z. and Leu, M.C. (1989) Design of Optical Triangulation Devices. Optics \& Laser Technology, 21, 339-341. http://dx.doi.org/10.1016/0030-3992(89)90068-6

[5] Donges, A. and Noll, R. (2015) Laser Measurement Technology: Fundamentals and Applications. Springer, Heidelberg. http://dx.doi.org/10.1007/978-3-662-43634-9

[6] Dorsch, R.G., Häusler, G. and Herrmann, J.M. (1994) Laser Triangulation: Fundamental Uncertainty in Distance Measurement. Applied Optics, 33, 1306-1314. http://dx.doi.org/10.1364/AO.33.001306

[7] Beyerer, J., León, F.P. and Frese, C. (2012) Automatische Sichtprüfung. Springer, Heiderlberg.

[8] Fisher, R.B. and Naidau, D.K. (1996) A Comparative Analysis of Algorithms for Determining the Peak Position of a Stripe to Sub-Pixel Accuracy. In: Sanz, J.L.C., Ed., Image Technologie: Advances in Image Processing, Multimedia and Machine Vision, Springer, Heidelberg, 385-404

[9] Mueller, T., Poesch, A. and Reithmeier, E. (2015) Measurement Uncertainty of Microscopic Laser Triangulation on 
Technical Surfaces. Microscopy and Microanalysis, 21, 1443-1454. http://dx.doi.org/10.1017/S1431927615015330

[10] Ayers, G.R. and Dainty, J.C. (1988) Interative Blind Deconvolution Method and Its Applications. Optics Letters, $13,7$. http://dx.doi.org/10.1364/OL.13.000547

[11] Levin, A., Weiss, Y., Durand, F. and Freeman, W.T. (2009) Understanding and Evaluating Blind Deconvolution Algorithms. IEEE Computer Society Conference on Computer Vision and Pattern Recognition Workshops (CVPR Workshops), Miami, 20-25 June 2009, 1964-1971.

[12] Kundur, D. and Hatzinakos, D. (1996) Blind Image Deconvolution. IEEE Signal Processing Magazine, 13, 43-64. http://dx.doi.org/10.1109/79.489268

[13] Wallace, W., Schaefer, L.H. and Swedlow, J.R. (2001) A Workingperson's Guide to Deconvolution in Light Microscopy. Biotechniques, 31, 1076-1097.

[14] Krishnan, D., Tay, T. and Fergus, R. (2011) Blind Deconvolution Using a Normalized Sparsity Measure. IEEE Conference on Computer Vision and Pattern Recognition (CVPR), Providence, 20-25 June 2011, 233-240. http://dx.doi.org/10.1109/cvpr.2011.5995521 